\title{
Selective formic acid dehydrogenation catalyzed by Fe-PNP pincer complexes based on the 2,6-diaminopyridine scaffold
}

\author{
Irene Mellone, ${ }^{\mathrm{a}}$ Nikolaus Gorgas, ${ }^{\mathrm{b}}$ Federica Bertini, ${ }^{\mathrm{a}}$ Maurizio Peruzzini, ${ }^{\mathrm{a}}$ Karl Kirchner, ${ }^{, \mathrm{b}}$ and \\ Luca Gonsalvi*,a \\ ${ }^{a}$ Consiglio Nazionale delle Ricerche (CNR), Istituto di Chimica dei Composti Organometallici (ICCOM), Via \\ Madonna del Piano 10, 50019 Sesto Fiorentino (Firenze), Italy. Email: 1.gonsalvi@iccom.cnr.it \\ ${ }^{\mathrm{b}}$ Institute of Applied Synthetic Chemistry, Vienna University of Technology, Getreidemarkt 9/163-AC, A-1060 Wien, \\ Austria. Email: karl.kirchner@tuwien.ac.at

\section{SUPPORTING INFORMATION}

1. Additional Tables and Reaction Profiles

2. DFT Calculations

3. References 


\section{ADDITIONAL TABLES AND REACTION PROFILES}

Table S1. Effect of the FA/NEt 3 ratio on the catalytic activity of $2 .{ }^{[a]}$

\begin{tabular}{|c|c|c|c|c|}
\hline Entry & $\mathrm{NEt}_{3}(\mathrm{~mol} \%)$ & $\operatorname{TOF}_{1 \mathrm{~h}}{ }^{[\mathrm{b}]}$ & TON $^{[c]}$ & conversion $(\%)$ \\
\hline 1 & 25 & 102 & $204(3)$ & 20 \\
\hline 2 & 50 & 276 & $653(3)$ & 65 \\
\hline 3 & 100 & 398 & $816(3)$ & 82 \\
\hline 4 & 200 & 418 & $827(3)$ & 83 \\
\hline
\end{tabular}
${ }^{[a]}$ Reaction conditions: $2(0.01 \mathrm{mmol})$; FA $(10 \mathrm{mmol})$; specified amount of $\mathrm{NEt}_{3}$, THF $(4.0 \mathrm{~mL}), 60{ }^{\circ} \mathrm{C}$. Gas evolution measured by manual gas
buret. ${ }^{\left[{ }^{b}\right]}$ Defined as $\mathrm{mmol}_{\mathrm{H} 2 \text { produced }} / \mathrm{mmol}_{\text {catalyst }} \mathrm{X} \mathrm{h}^{-1}$ (calculated after $\left.1 \mathrm{~h}\right) .{ }^{[c]}$ Defined as $\mathrm{mmol}_{\mathrm{H} 2 \text { produced }} / \mathrm{mmol}_{\text {catalyst. }}$ Run time (h) in parenthesis. All tests were repeated at least twice to check for reproducibility (error $\pm 10 \%$ ).

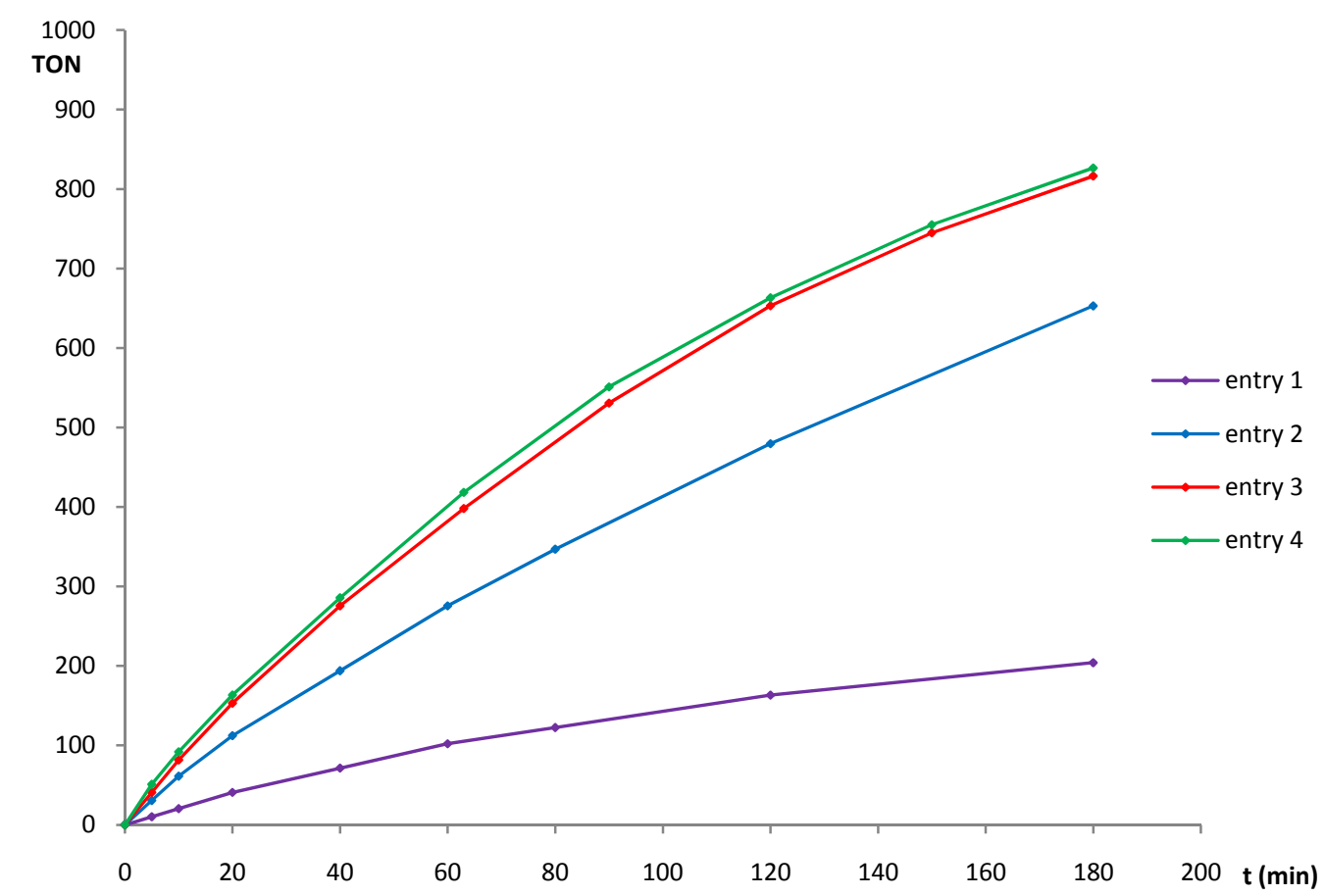

Figure S1. Reaction profiles for FA/NEt $t_{3}$ ratio effect screening in FA dehydrogenation with 2. 
Table S2. Effect of different solvents on the catalytic activity of $2 .^{[a]}$

\begin{tabular}{|c|c|c|c|c|}
\hline Entry & solvent & $\operatorname{TOF}_{1 \mathrm{~h}}{ }^{|b|}$ & TON $\mathbf{N}^{|c|}$ & conversion $(\%)$ \\
\hline 1 & THF & 612 & $1000(3)$ & 100 \\
\hline 2 & $\mathrm{PC}$ & 500 & $1000(3)$ & 100 \\
\hline 3 & dioxane & 378 & $878(3)$ & 88 \\
\hline 4 & $\mathrm{EtOH}$ & 165 & $650(3)$ & 65 \\
\hline
\end{tabular}

${ }^{[a]}$ Reaction conditions: 2 (0.01 mmol); FA $(10 \mathrm{mmol})$; $\mathrm{NEt}_{3}(100 \mathrm{~mol} \%)$, solvent $(2.0 \mathrm{~mL}), 60^{\circ} \mathrm{C}$. Gas evolution measured by manual gas buret. ${ }^{[b]}$

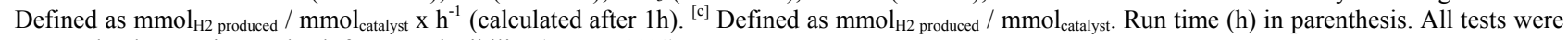
repeated at least twice to check for reproducibility (error $\pm 10 \%$ ).

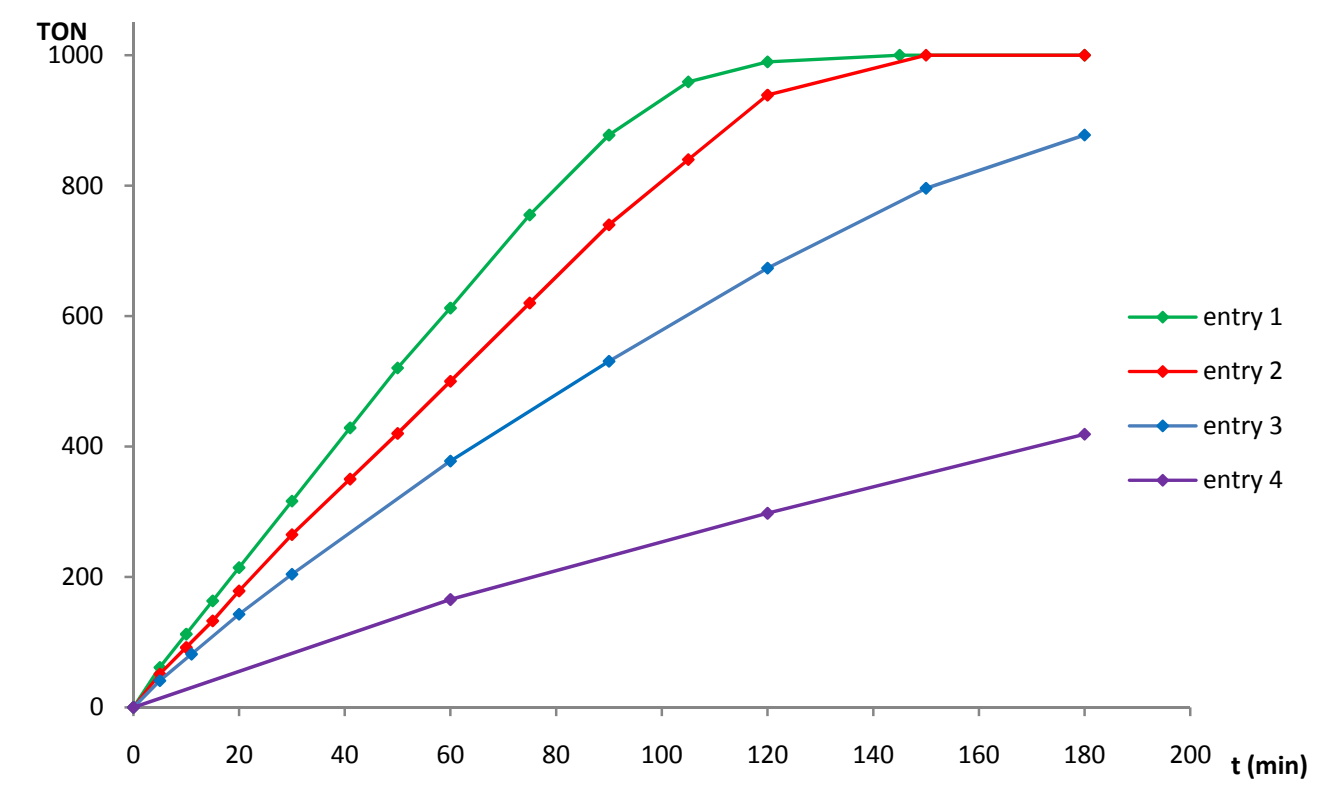

Figure S2. Reaction profiles for solvent effect screening in FA dehydrogenation with 2. 
Table S3. Effect of different amines on the catalytic activity of $2 .^{[a]}$

\begin{tabular}{ccccc}
\hline Entry & amine (mol\%) & TOF $_{\mathbf{1 h}}{ }^{|b|}$ & TON $^{|c|}$ & conv. (\%) \\
\hline $\mathbf{1}$ & $\operatorname{NEt}_{3}(50)$ & 593 & $980(3)$ & 98 \\
$\mathbf{2}$ & $\operatorname{DMOA}(50)$ & 673 & $980(3)$ & 98 \\
$\mathbf{3}$ & $\operatorname{DBU}(50)$ & 459 & $571(3)$ & 57 \\
\hline
\end{tabular}

${ }^{[a]}$ Reaction conditions: 2 ( $\left.0.01 \mathrm{mmol}\right)$; FA $(10 \mathrm{mmol})$; specified amine $(50 \mathrm{~mol} \%)$, THF $(2.0 \mathrm{~mL}), 60^{\circ} \mathrm{C}$. Gas evolution measured by manual gas buret. ${ }^{[b]}$ Defined as $\mathrm{mmol}_{\mathrm{H} 2 \text { produced }} / \mathrm{mmol}_{\text {catalyst }} \mathrm{x} \mathrm{h}^{-1}$ (calculated after $\left.1 \mathrm{~h}\right) .{ }^{[\mathrm{cc}}$ Defined as $\mathrm{mmol}_{\mathrm{H} 2 \text { produced }} / \mathrm{mmol}_{\text {catalyst. }}$ Run time (h) in parenthesis. All tests were repeated at least twice to check for reproducibility (error $\pm 10 \%$ ).

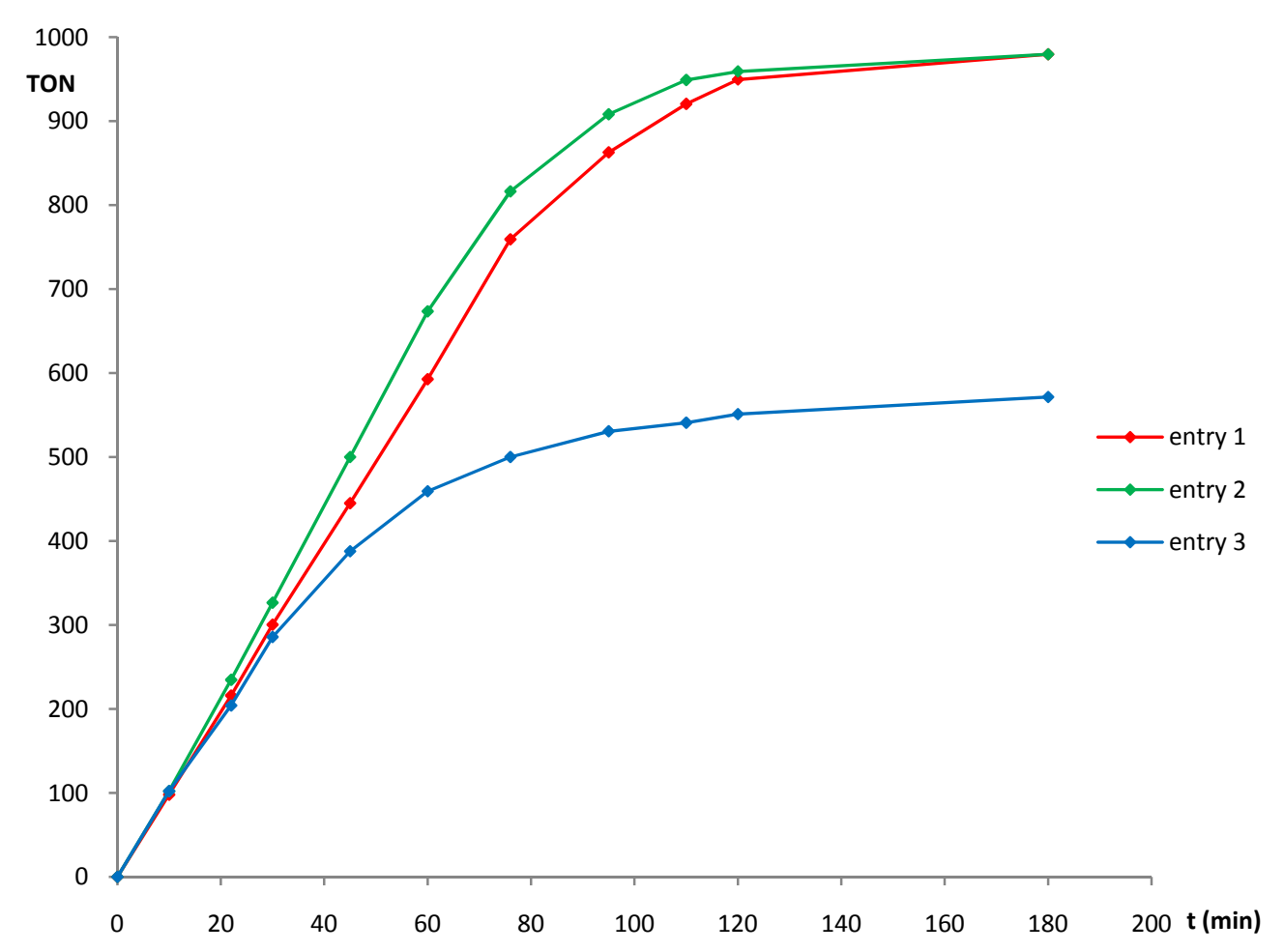

Figure S3. Reaction profiles for amine effect screening in FA dehydrogenation with 2. 
Table S4. Effect of the reaction temperature on the catalytic activity of 2.

\begin{tabular}{|c|c|c|c|c|c|}
\hline Entry & Solvent & $\mathrm{T}\left({ }^{\circ} \mathrm{C}\right)$ & $\operatorname{TOF}_{1 \mathrm{~h}}^{[b]}$ & TON $^{|c|}$ & conv. $(\%)$ \\
\hline 1 & THF & 40 & 79 & $180(3)$ & 18 \\
\hline 2 & THF & 60 & 612 & $1000(3)$ & 100 \\
\hline 3 & $\mathrm{PC}$ & 60 & 500 & $1000(3)$ & 100 \\
\hline 4 & $\mathrm{PC}$ & 80 & $1800^{[\mathrm{d}]}$ & $1000(0.6)$ & 100 \\
\hline
\end{tabular}

${ }^{[a]}$ Reaction conditions: 2 (0.01 mmol); FA $(10 \mathrm{mmol})$; $\mathrm{NEt}_{3}(100 \mathrm{~mol} \%)$, solvent $(2.0 \mathrm{~mL})$. Gas evolution measured by manual gas buret. ${ }^{\left[{ }^{b}\right]}$ Defined as $\mathrm{mmol}_{\mathrm{H} 2 \text { produced }} / \mathrm{mmol}_{\text {catalyst }} \times \mathrm{h}^{-1}$ (calculated after $1 \mathrm{~h}$ ). ${ }^{[\mathrm{c}]}$ Defined as $\mathrm{mmol}_{\mathrm{H} 2 \text { produced }} / \mathrm{mmol}_{\text {catalyst. }}$ Run time (h) in parenthesis. ${ }^{[\mathrm{d}]} \mathrm{TOF}$ calculated after $20 \mathrm{~min}$ due to fast reaction. All tests were repeated at least twice to check for reproducibility (error $\pm 10 \%$ ).

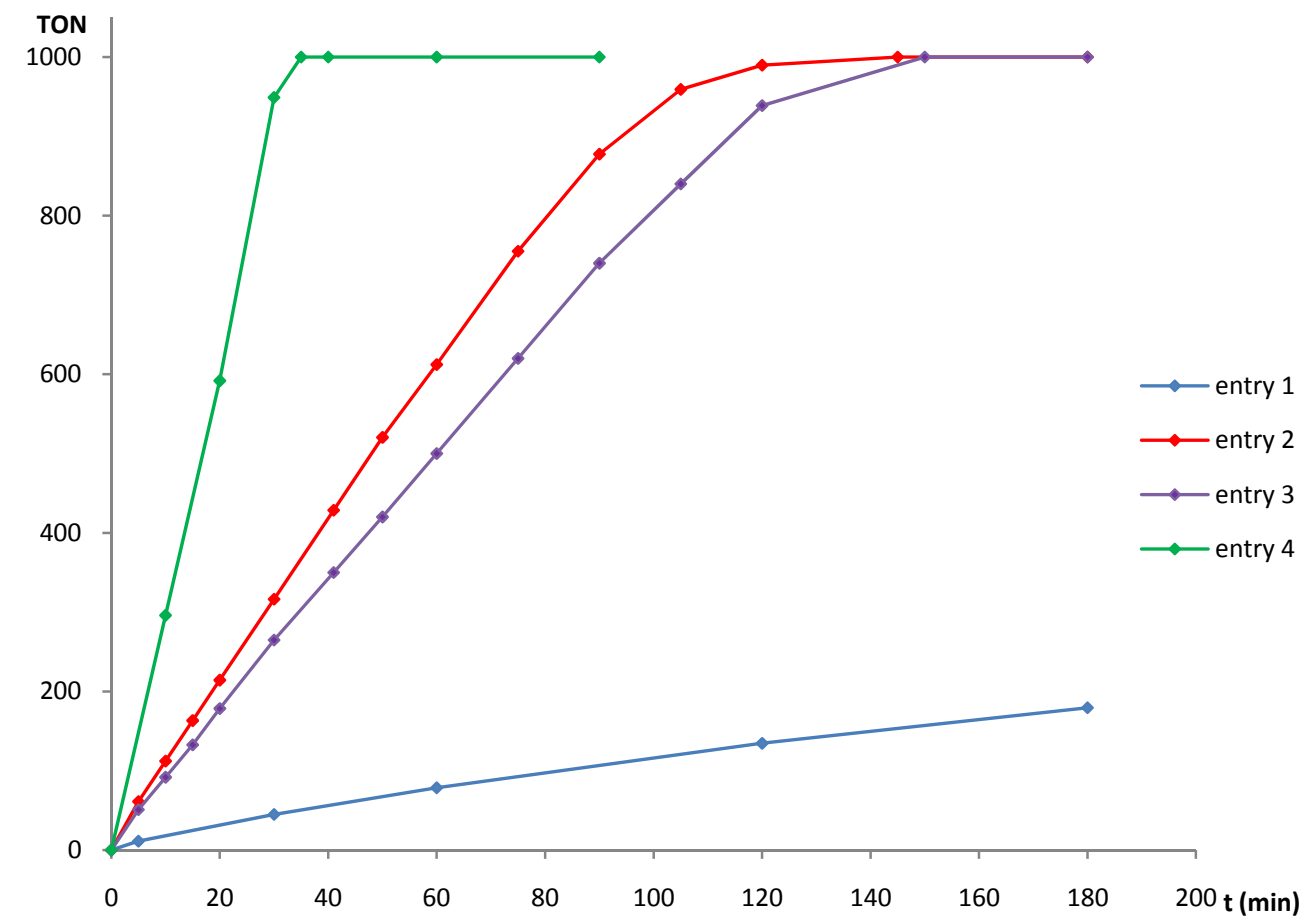

Figure S4. Reaction profiles for temperature effect screening in FA dehydrogenation with 2. 
Table S5. Effect of the substrate concentration on the catalytic activity of $\mathbf{2}$.

\begin{tabular}{|c|c|c|c|c|}
\hline Entry & {$[\mathrm{FA}](\mathrm{mol} / \mathrm{L})$} & $\mathrm{TOF}_{1 \mathrm{~h}}^{[\mathrm{d}]}$ & TON $^{[\mathrm{el}}$ & conversion $(\%)$ \\
\hline $1^{[a]}$ & 2.5 & 398 & $816(3)$ & 82 \\
\hline $2^{[b]}$ & 5.0 & 612 & $1000(2.5)$ & 100 \\
\hline $3^{|c|}$ & 10.0 & 770 & $1000(2)$ & 100 \\
\hline
\end{tabular}

Reaction conditions: ${ }^{\left[{ }^{a}\right]} 2(0.01 \mathrm{mmol})$; FA $(10 \mathrm{mmol}) ; \mathrm{NEt}_{3}(100 \mathrm{~mol} \%)$, THF $(4.0 \mathrm{~mL}), 60^{\circ} \mathrm{C} .{ }^{[\mathrm{b}]} \mathrm{THF}(2.0 \mathrm{~mL}) .{ }^{[\mathrm{c}]} \mathrm{THF}(1.0 \mathrm{~mL})$. Gas evolution measured by manual gas buret. ${ }^{[\mathrm{d}]}$ Defined as $\mathrm{mmol}_{\mathrm{H} 2 \text { produced }} / \mathrm{mmol}_{\text {catalyst }} \mathrm{x} \mathrm{h}^{-1}$ (calculated after $1 \mathrm{~h}$ ). ${ }^{\left[{ }^{[\mathrm{e}]}\right.}$ Defined as $\mathrm{mmol}_{\mathrm{H} 2 \text { produced }} / \mathrm{mmol}_{\text {catalyst }}$ Run time (h) in parenthesis. All tests were repeated at least twice to check for reproducibility (error $\pm 10 \%$ ).

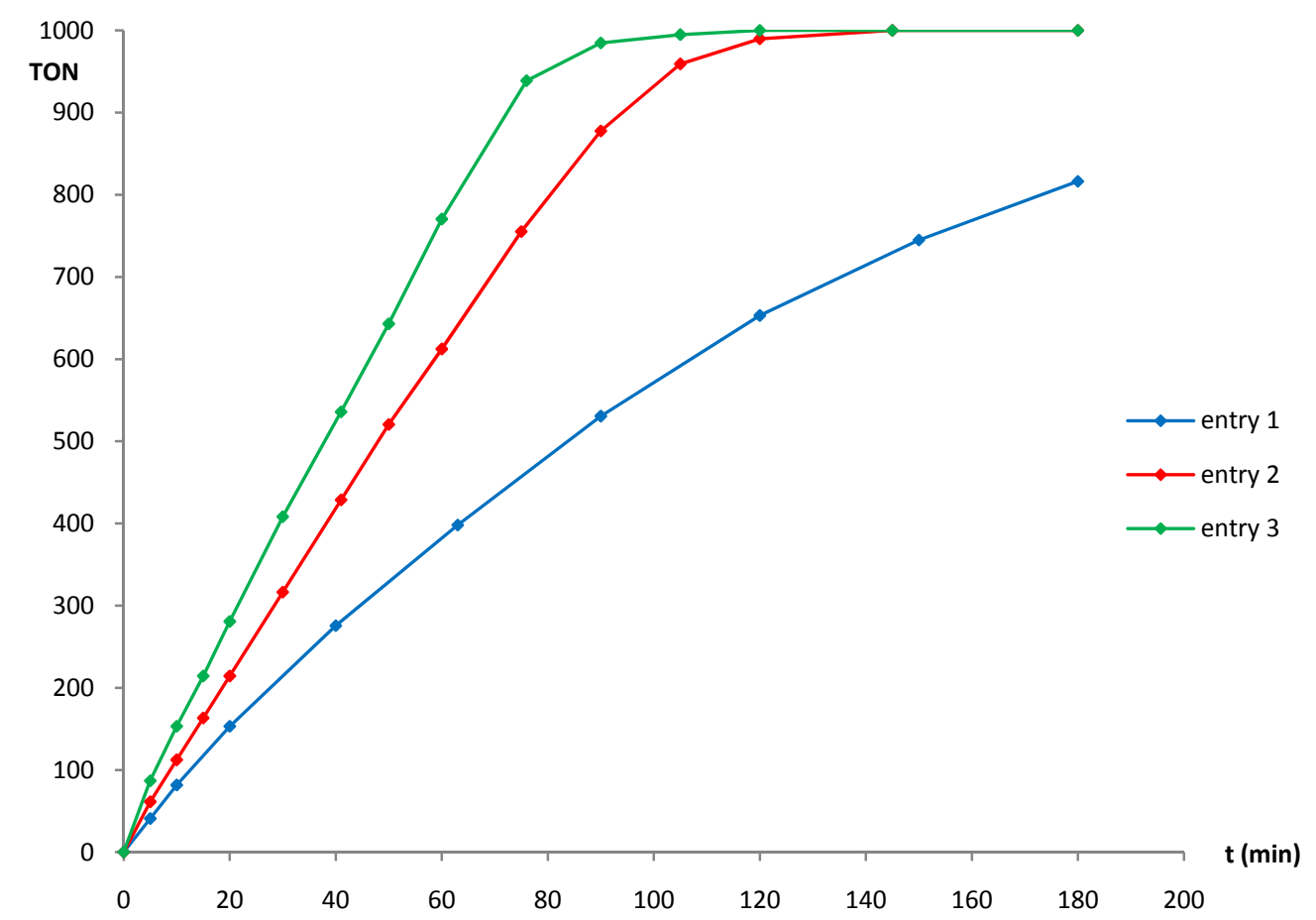

Figure S5. Reaction profiles for substrate concentration effect screening in FA dehydrogenation with $\mathbf{2}$. 
Table S6. Effect of catalyst loading on the catalytic activity of 2.

\begin{tabular}{|c|c|c|c|c|c|c|}
\hline Entry & {$[\mathrm{FA}](\mathrm{mol} / \mathrm{L})$} & solvent & $\mathbf{T}\left({ }^{\circ} \mathbf{C}\right)$ & $\mathrm{TOF}_{1 \mathrm{~h}}^{[\mathrm{d}]}$ & TON $^{[\mathrm{el}}$ & conversion $(\%)$ \\
\hline $1^{[a]}$ & 10.0 & THF & 60 & 918 & $2245(6)$ & 22 \\
\hline $2^{[b]}$ & 5.0 & $\mathrm{PC}$ & 80 & 1714 & $6286(6)$ & 63 \\
\hline $3^{[\mathrm{cc}]}$ & 10.0 & $\mathrm{PC}$ & 80 & 2635 & $10000(6)$ & 100 \\
\hline
\end{tabular}

Reaction conditions: ${ }^{[\mathrm{a}]} 2(0.005 \mathrm{mmol}) ; \mathrm{FA}(50 \mathrm{mmol}) ; \mathrm{NEt}_{3}(100 \mathrm{~mol} \%)$, THF $(5.0 \mathrm{~mL}), 60{ }^{\circ} \mathrm{C} .{ }^{[b]} \mathbf{2}(0.005 \mathrm{mmol}) ; \mathrm{FA}\left(50 \mathrm{mmol}^{\circ}\right) ; \mathrm{NEt}_{3}(100$ mol\%), PC $(10.0 \mathrm{~mL}), 80^{\circ} \mathrm{C}$. ${ }^{[c]} \mathbf{2}(0.005 \mathrm{mmol}) ; \mathrm{FA}(50 \mathrm{mmol}) ; \mathrm{NEt}_{3}(100 \mathrm{~mol} \%)$, PC $(5.0 \mathrm{~mL}), 80^{\circ} \mathrm{C}$. Gas evolution measured by manual gas buret. ${ }^{[\mathrm{d}]}$ Defined as $\mathrm{mmol}_{\mathrm{H} 2 \text { produced }} / \mathrm{mmol}_{\text {catalyst }} \mathrm{X} \mathrm{h}^{-1}$ (calculated after $\left.1 \mathrm{~h}\right) .{ }^{[\mathrm{e}]}$ Defined as $\mathrm{mmol}_{\mathrm{H} 2 \text { produced }} / \mathrm{mmol}_{\text {catalyst. }}$ Run time (h) in parenthesis. All tests were repeated at least twice to check for reproducibility (error $\pm 10 \%$ ).

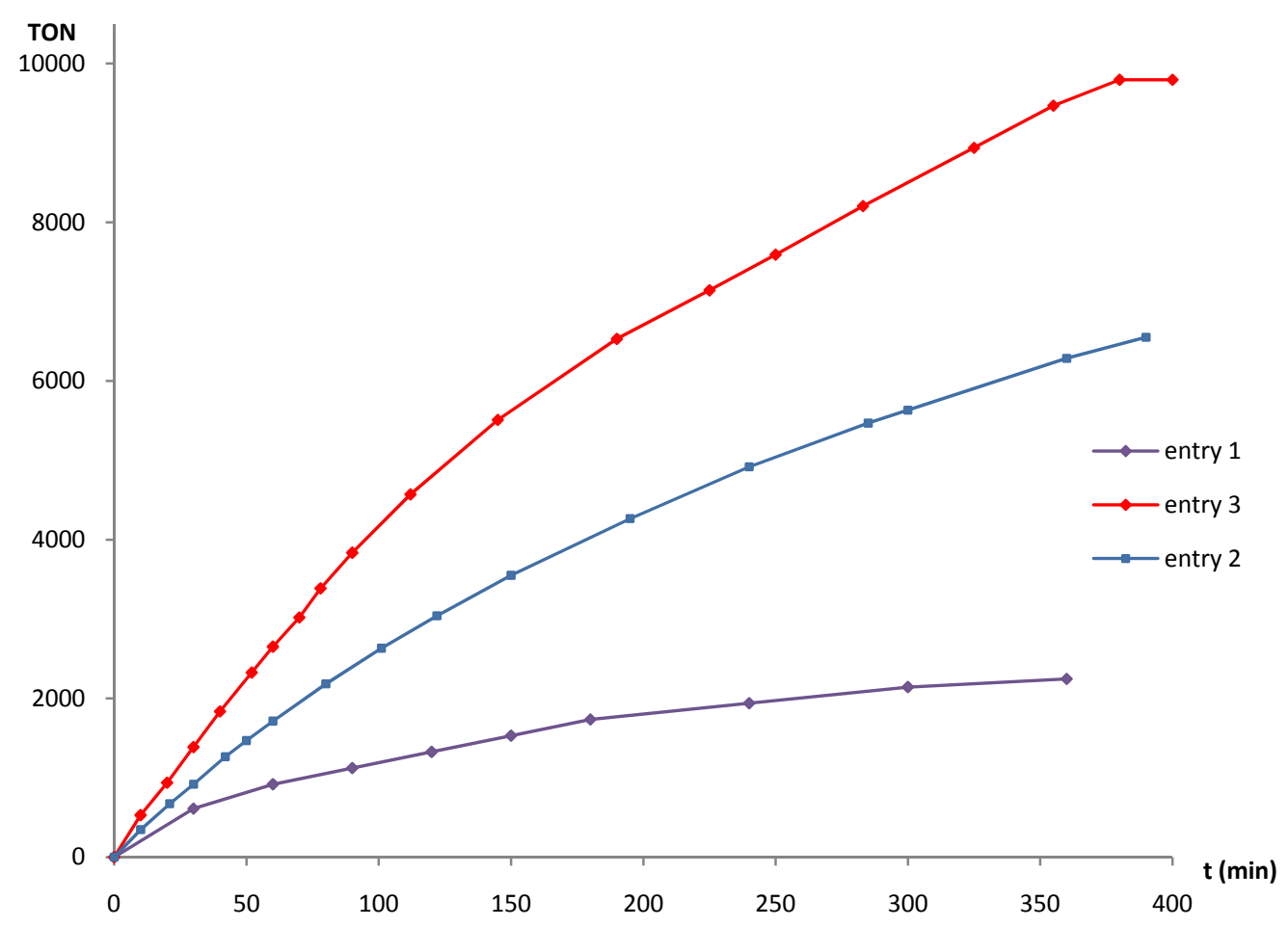

Figure S6. Reaction profiles for catalyst loading effect screening in FA dehydrogenation with 2. 
Table S7. Slow FA addition tests in the presence of $\mathbf{2}$ and $\mathbf{3}$ and $\mathrm{NEt}_{3}$.

\begin{tabular}{|c|c|c|c|c|c|}
\hline Entry & No. run & Initial FA/cat & $\mathrm{TOF}_{10 \min }{ }^{[\mathrm{d}]}$ & TON $^{[\mathrm{el}}$ & conversion $(\%)^{[\mathrm{f}]}$ \\
\hline \multirow[t]{5}{*}{$1^{[a]}$} & 1 & 5000 & 2574 & 2500 & $50(65)$ \\
\hline & 2 & & 2628 & 2500 & $50(135)$ \\
\hline & 3 & & 2439 & 2500 & $50(230)$ \\
\hline & 4 & & 2140 & 2500 & $50(350)$ \\
\hline & 5 & & 1874 & 2170 & $47(520)$ \\
\hline \multirow[t]{12}{*}{$2^{[b]}$} & 1 & 1000 & 1782 & 502 & $50(17)$ \\
\hline & 2 & & 1715 & 502 & $50(35)$ \\
\hline & 3 & & 1668 & 502 & $50(52)$ \\
\hline & 4 & & 1795 & 502 & $50(70)$ \\
\hline & 5 & & 1727 & 502 & $50(87)$ \\
\hline & 6 & & 1701 & 502 & $50(105)$ \\
\hline & 7 & & 1724 & 502 & $50(122)$ \\
\hline & 8 & & 1710 & 502 & $50(145)$ \\
\hline & 9 & & 1616 & 502 & $50(175)$ \\
\hline & 10 & & 1517 & 502 & $50(210)$ \\
\hline & 11 & & 1401 & 502 & $50(250)$ \\
\hline & 12 & & 1279 & 52 & $5(270)$ \\
\hline \multirow[t]{5}{*}{$3^{[\mathrm{cl}}$} & 1 & 5000 & 2844 & 2500 & $50(60)$ \\
\hline & 2 & & 2664 & 2500 & $50(130)$ \\
\hline & 3 & & 2494 & 2500 & $50(225)$ \\
\hline & 4 & & 2141 & 2500 & $50(345)$ \\
\hline & 5 & & 1882 & 2300 & $48(550)$ \\
\hline
\end{tabular}

Reaction conditions: ${ }^{\text {[a] }} 2(0.005 \mathrm{mmol})$; FA $(25 \mathrm{mmol}$, initial $) ; \mathrm{NEt}_{3}(100 \mathrm{~mol} \%), \mathrm{PC}(5.0 \mathrm{~mL}), 80{ }^{\circ} \mathrm{C} .{ }^{[b]} \mathbf{2}(0.01 \mathrm{mmol}) ; \mathrm{FA}(10 \mathrm{mmol}$, initial $) ; \mathrm{NEt}$ $(100 \mathrm{~mol} \%), \mathrm{PC}(5.0 \mathrm{~mL}), 80^{\circ} \mathrm{C} .:{ }^{[\mathrm{cc}]} 3(0.005 \mathrm{mmol}) ; \mathrm{FA}(25 \mathrm{mmol}$, initial $) ; \mathrm{NEt}_{3}(100 \mathrm{~mol} \%), \mathrm{PC}(5.0 \mathrm{~mL}), 80^{\circ} \mathrm{C}$. ${ }^{[\mathrm{d}]}$ Defined as mmol $\mathrm{H}_{\mathrm{H}}$ produced $/$ $\mathrm{mmol}_{\text {catalyst }} \mathrm{X} \mathrm{h}^{-1}$ (calculated after $\left.10 \mathrm{~min}\right)$. ${ }^{\left[{ }^{[}\right]}$Defined as $\mathrm{mmol}_{\mathrm{H} 2 \text { produced }} / \mathrm{mmol}_{\text {catalyst }}{ }^{[\mathrm{f}]}$ Run time (min) in parenthesis. All tests were repeated at least twice to check for reproducibility (error $\pm 10 \%$ ) 


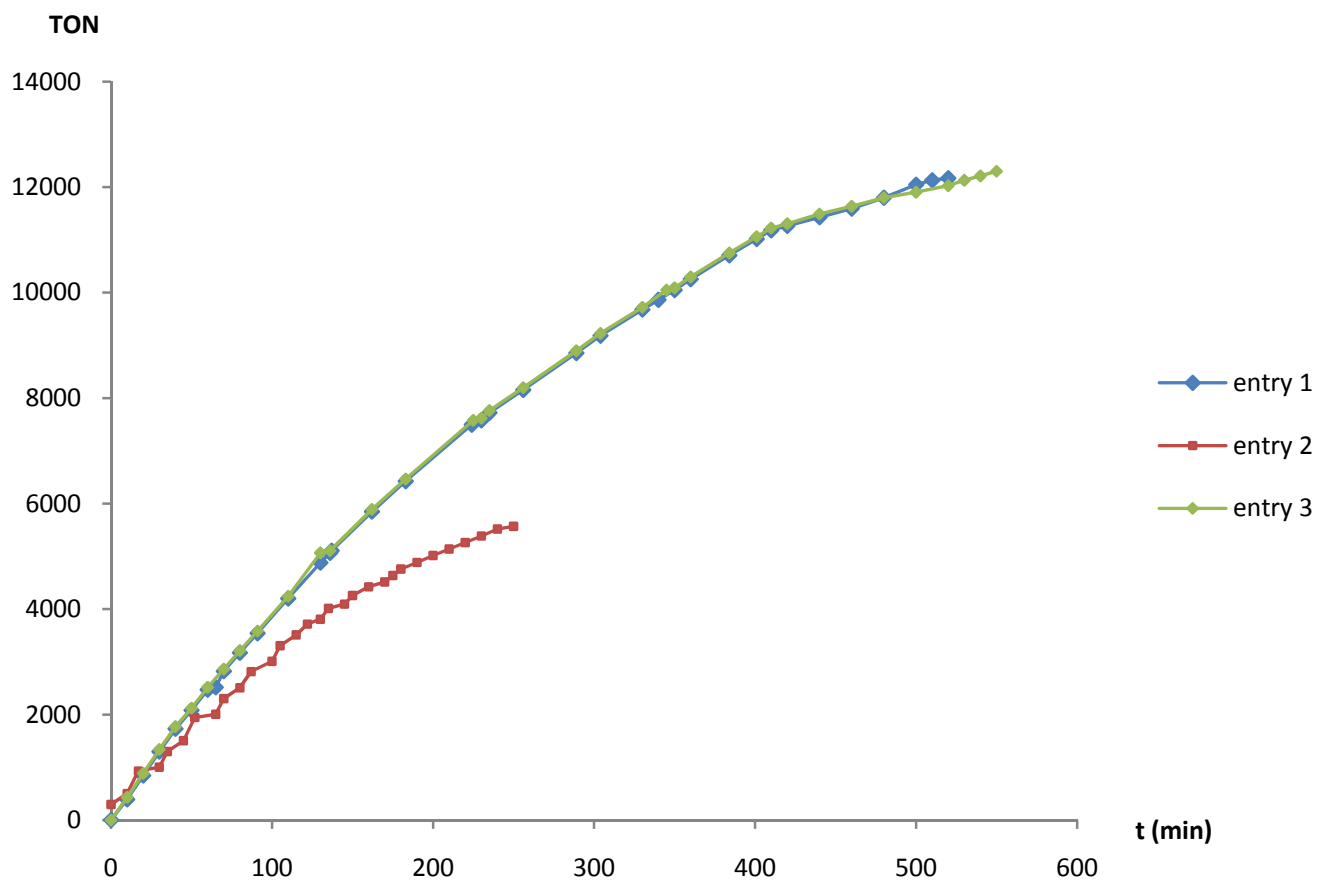

Figure S4. Reaction profiles for slow feed FA dehydrogenation with 2 and 3. Conditions: Neat FA (12.5 mmol aliquots) added after 65, 135, 230, 350 min (entry 1); 17, 35, 52, 70, 87, 105, 122, 145, 175, 210, 250 min (entry 2); 60, 130, 225, 345 min (entry 3).

\section{COMPUTATIONAL DETAILS}

Calculations were performed using the GAUSSIAN 09 software package, ${ }^{1}$ without symmetry constraints. The optimized geometries were obtained with the B3LYP functional. ${ }^{2}$ That functional includes a mixture of Hartree-Fock ${ }^{3}$ exchange with DFT ${ }^{4}$ exchange-correlation, given by Becke's three parameter functional with the Lee, Yang and Parr correlation functional, which includes both local and non-local terms. The basis set used consists of the Stuttgart/Dresden ECP (SDD) basis set ${ }^{5}$ to describe the electrons of the iron atom, and a standard 6-31g $(\mathrm{d}, \mathrm{p})$ basis set ${ }^{6}$ for all other atoms. Frequency calculations were performed to confirm the nature of the stationary points yielding no imaginary frequency for the the minima. ${ }^{1} \mathrm{H}$ chemical sifts were calculated at the B3LYP level of theory for the optimized structures of trans$\left[\mathrm{Fe}\left(\mathrm{PNP}^{\mathrm{Me}}-i \mathrm{Pr}\right)(\mathrm{H})(\mathrm{CO})\left(\eta^{1}-\mathrm{OCOH}\right)\right]$ (4) and trans-[Fe( $\left.\left.\mathrm{PNP}^{\mathrm{Me}}-i \mathrm{Pr}\right)(\mathrm{H})(\mathrm{CO})\left(\eta^{1}-\mathrm{HCOOH}\right)\right]^{+}$(5) using the gaugeindependent atomic orbital (GIAO) method in Gaussian 09 with the above basis sets. Chemical shifts are given with respect to $\mathrm{Si}\left(\mathrm{Me}_{3}\right)_{4}$ (TMS) at the same computational level. ${ }^{7}$

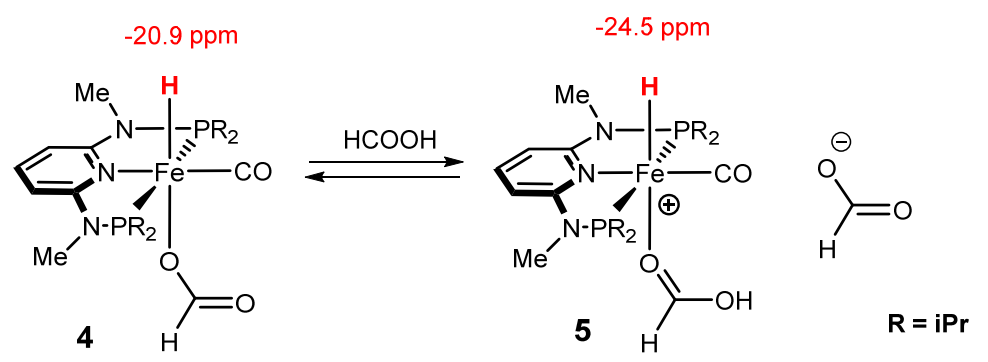

Scheme S1. Calculated ${ }^{1} \mathrm{H}$ NMR hydride shifts for trans-[Fe( $\left.\left.\mathrm{PNP}^{\mathrm{Me}}-i \mathrm{Pr}\right)(\mathrm{H})(\mathrm{CO})\left(\eta^{1}-\mathrm{OCOH}\right)\right](4)$ and trans-[Fe( $\mathrm{PNP}^{\mathrm{Me}}$ $\left.i \operatorname{Pr})(\mathrm{H})(\mathrm{CO})\left(\eta^{1}-\mathrm{HCOOH}\right)\right]^{+}(\mathbf{5})$. 


\begin{tabular}{|c|c|c|c|}
\hline 26 & -0.006874000 & -0.467660000 & 0.118446000 \\
\hline 15 & -2.210393000 & -0.270927000 & 0.197924000 \\
\hline 15 & 2.215235000 & -0.187877000 & 0.143634000 \\
\hline 7 & -0.041874000 & 1.573026000 & 0.082817000 \\
\hline 7 & -2.377999000 & 1.471292000 & 0.235813000 \\
\hline 7 & 2.302602000 & 1.553367000 & -0.045780000 \\
\hline 6 & -1.226375000 & 2.237750000 & 0.123117000 \\
\hline 6 & -1.288876000 & 3.639850000 & 0.054730000 \\
\hline 6 & -0.097776000 & 4.345099000 & -0.040043000 \\
\hline 6 & 1.119221000 & 3.681482000 & -0.067230000 \\
\hline 6 & 1.116635000 & 2.274929000 & -0.012370000 \\
\hline 6 & -3.216772000 & -0.816056000 & -1.308050000 \\
\hline 6 & -4.747554000 & -0.845435000 & 956000 \\
\hline 6 & -2.692433000 & -2.160806000 & -1.849865000 \\
\hline 6 & -3.201688000 & -0.807331000 & 1.701011000 \\
\hline 6 & -3.163294000 & -2.336957000 & 30000 \\
\hline 6 & -2.718374000 & -0.103400000 & 2.977 \\
\hline 6 & 3.103711000 & -0.949703000 & 00000 \\
\hline 6 & 4.378752000 & -0.296750000 & -1.879636000 \\
\hline 6 & 3.311835000 & 720000 & 7000 \\
\hline 6 & 3.166040000 & -0.505628000 & 1.75 \\
\hline 6 & 2.702448000 & -1.816275000 & 2.414191000 \\
\hline 6 & 4.704014000 & -0.450335000 & 2000 \\
\hline 1 & -2.235289000 & 4.159777000 & 26000 \\
\hline 1 & -0.118700000 & 5.429621000 & 1000 \\
\hline 1 & 47000 & 4.23 & 9000 \\
\hline 1 & -2.947694000 & -0.045318000 & 02000 \\
\hline 1 & -5.191262000 & -1.120028000 & -2.129614000 \\
\hline 1 & -5.177085000 & 0.117840000 & -0.879 \\
\hline 1 & -5.076135000 & -1.594496000 & -0.43 \\
\hline 1 & -3.220916000 & -2.405297000 & -2.778 \\
\hline 1 & -2.867653000 & -2.984715000 & -1.150 \\
\hline 1 & -1.625037000 & -2.100647000 & -2.067809000 \\
\hline 1 & -4.244181000 & -0.516538000 & 1.518401000 \\
\hline 1 & -3.774275000 & -2.624062000 & 2000 \\
\hline 1 & -2.145493000 & -2.693205000 & 0000 \\
\hline 1 & -3.556678000 & -2.867483000 & 0.9988 \\
\hline 1 & -3.336280000 & -0.418036000 & 3.826616000 \\
\hline 1 & -2.782701000 & 5463000 & 98000 \\
\hline 1 & -1.677655000 & -0.360115000 & 3.192637000 \\
\hline 1 & 2.315366000 & -0.846229000 & 6000 \\
\hline 1 & 4.702571000 & -0.863198000 & -2.760337000 \\
\hline 1 & 5.211354000 & -0.302972000 & -1.168280000 \\
\hline 1 & 4.202606000 & 0.728162000 & -2.212532000 \\
\hline 1 & 3.601673000 & -2.927572000 & -2.036373000 \\
\hline 1 & 2.402786000 & -2.959514000 & -0.748164000 \\
\hline 1 & 4.109859000 & -2.662820000 & -0.367971000 \\
\hline 1 & 2.800958000 & 0.321545000 & 2.374446000 \\
\hline 1 & 3.167813000 & -1.905737000 & 3.402692000 \\
\hline 1 & 2.996090000 & -2.695758000 & 1.833673000 \\
\hline 1 & 1.619728000 & -1.835305000 & 2.543653000 \\
\hline 1 & 5.091081000 & -0.596968000 & 2.730644000 \\
\hline 1 & 5.097544000 & 0.503018000 & 1.358008000 \\
\hline 1 & 5.124347000 & -1.245631000 & 1.093587000 \\
\hline 1 & -0.000904000 & -0.379224000 & 1.635835000 \\
\hline 6 & 0.020091000 & -2.208455000 & 0.323813000 \\
\hline
\end{tabular}




$\begin{array}{rrrr}8 & 0.037472000 & -3.352070000 & 0.530918000 \\ 6 & 3.549738000 & 2.318311000 & -0.095755000 \\ 1 & 3.668952000 & 2.949781000 & 0.794069000 \\ 1 & 4.393026000 & 1.638842000 & -0.150263000 \\ 1 & 3.582296000 & 2.956964000 & -0.985531000 \\ 6 & -3.659951000 & 2.161584000 & 0.358463000 \\ 1 & -3.938700000 & 2.676911000 & -0.569411000 \\ 1 & -4.439349000 & 1.440518000 & 0.595047000 \\ 1 & -3.632482000 & 2.896404000 & 1.170974000 \\ 8 & 0.118353000 & -0.521569000 & -1.937601000 \\ 6 & -0.052722000 & 0.418675000 & -2.812792000 \\ 8 & 0.512539000 & 0.508727000 & -3.896862000 \\ 1 & -0.800926000 & 1.200885000 & -2.525457000\end{array}$

$\begin{array}{lrrr}\text { trans }-\left[\mathrm{Fe}\left(\mathrm{PNP}{ }^{\mathrm{Me}}-\mathrm{Pr}\right)(\mathrm{H})(\mathrm{CO})\left(\eta^{1}-\mathrm{HCOOH}\right)\right]^{+}(\mathbf{5}) \\ 26 & 0.054929000 & -0.511116000 & -0.003161000 \\ 15 & 2.272352000 & -0.321273000 & -0.187763000 \\ 15 & -2.171597000 & -0.299731000 & -0.220593000 \\ 7 & 0.063746000 & 1.489437000 & -0.334507000 \\ 7 & 2.404271000 & 1.394956000 & -0.507797000 \\ 7 & -2.289573000 & 1.450788000 & -0.275617000 \\ 6 & 1.243904000 & 2.152704000 & -0.507473000 \\ 6 & 1.282284000 & 3.547395000 & -0.675396000 \\ 6 & 0.080271000 & 4.239705000 & -0.709618000 \\ 6 & -1.130493000 & 3.571897000 & -0.584623000 \\ 6 & -1.111150000 & 2.178634000 & -0.401182000 \\ 6 & 3.344302000 & -0.593449000 & 1.349106000 \\ 6 & 4.868979000 & -0.614493000 & 1.142196000 \\ 6 & 2.889746000 & -1.846720000 & 2.124122000 \\ 6 & 3.189272000 & -1.107196000 & -1.620163000 \\ 6 & 3.156570000 & -2.643940000 & -1.526767000 \\ 6 & 2.652799000 & -0.625273000 & -2.976745000 \\ 6 & -3.178870000 & -0.863414000 & 1.273030000 \\ 6 & -4.503369000 & -0.158096000 & 1.609654000 \\ 6 & -3.370484000 & -2.393936000 & 1.235168000 \\ 6 & -2.963313000 & -0.891499000 & -1.829299000 \\ 6 & -2.461758000 & -2.297015000 & -2.216013000 \\ 6 & -4.497270000 & -0.827810000 & -1.946511000 \\ 1 & 2.220089000 & 4.072832000 & -0.777069000 \\ 1 & 0.085791000 & 5.317186000 & -0.843450000 \\ 1 & -2.059533000 & 4.118648000 & -0.634725000 \\ 1 & 3.094092000 & 0.285515000 & 1.959473000 \\ 1 & 5.360865000 & -0.716899000 & 2.115233000 \\ 1 & 5.256873000 & 0.297036000 & 0.682408000 \\ 1 & 5.184407000 & -1.467141000 & 0.533423000 \\ 1 & 3.453709000 & -1.917120000 & 3.060092000 \\ 1 & 3.080266000 & -2.766020000 & 1.562878000 \\ 1 & 1.827458000 & -1.814834000 & 2.372172000 \\ 1 & 4.234132000 & -0.788468000 & -1.525707000 \\ 1 & 3.737866000 & -3.066045000 & -2.352706000 \\ 1 & 2.137120000 & -3.028627000 & -1.610028000 \\ 1 & 3.589160000 & -3.023477000 & -0.597440000 \\ 1 & 3.261757000 & -1.055561000 & -3.778562000 \\ 1 & 2.687154000 & 0.462765000 & -3.078008000 \\ 1 & 1.618189000 & -0.944487000 & -3.127305000 \\ 1 & -2.475790000 & -0.649045000 & 2.086481000 \\ 1 & -4.910314000 & -0.601069000 & 2.525336000 \\ 1 & -5.259826000 & -0.283965000 & 0.829973000\end{array}$




$\begin{array}{rrr}-4.372176000 & 0.909134000 & 1.801809000 \\ -3.732144000 & -2.729469000 & 2.212366000 \\ -2.445156000 & -2.935786000 & 1.028203000 \\ -4.116369000 & -2.693072000 & 0.493803000 \\ -2.527709000 & -0.180319000 & -2.544402000 \\ -2.834147000 & -2.542309000 & -3.215917000 \\ -2.828316000 & -3.068462000 & -1.533493000 \\ -1.372628000 & -2.345745000 & -2.241125000 \\ -4.786174000 & -1.144495000 & -2.954202000 \\ -4.906062000 & 0.173077000 & -1.799231000 \\ -4.988602000 & -1.507068000 & -1.244943000 \\ 0.083220000 & -0.736003000 & -1.473164000 \\ 0.052261000 & -2.269434000 & 0.156737000 \\ 0.053354000 & -3.426185000 & 0.192756000 \\ -3.548751000 & 2.182841000 & -0.470002000 \\ -3.593501000 & 2.642293000 & -1.464222000 \\ -4.385172000 & 1.501091000 & -0.368538000 \\ -3.665922000 & 2.966517000 & 0.285865000 \\ 3.668435000 & 2.063354000 & -0.836560000 \\ 3.999153000 & 2.730848000 & -0.032150000 \\ 4.443687000 & 1.318423000 & -1.000378000 \\ 3.569792000 & 2.644481000 & -1.758845000 \\ -0.031931000 & -0.094836000 & 2.119843000 \\ 0.054615000 & 0.919707000 & 2.785575000 \\ -0.073624000 & 0.872732000 & 4.105724000 \\ 0.236104000 & 1.910979000 & 2.343589000 \\ 0.019773000 & 1.755013000 & 4.497466000\end{array}$

\section{REFERENCES}

1 Gaussian 09, Revision A.02, Frisch, M. J.; Trucks, G. W.; Schlegel, H. B.; Scuseria, G. E.; Robb, M. A.; Cheeseman, J. R.; Scalmani, G.; Barone, V.; Mennucci, B.; Petersson, G. A.; Nakatsuji, H.; Caricato, M.; Li, X.; Hratchian, H. P.; Izmaylov, A. F.; Bloino, J.; Zheng, G.; Sonnenberg, J. L.; Hada, M.; Ehara, M.; Toyota, K.; Fukuda, R.; Hasegawa, J.; Ishida, M.; Nakajima, T.; Honda, Y.; Kitao, O.; Nakai, H.; Vreven, T.; Montgomery, Jr., J. A.; Peralta, J. E.; Ogliaro, F.; Bearpark, M.; Heyd, J. J.; Brothers, E.; Kudin, K. N.; Staroverov, V. N.; Kobayashi, R.; Normand, J.; Raghavachari, K.; Rendell, A.; Burant, J. C.; Iyengar, S. S.; Tomasi, J.; Cossi, M.; Rega, N.; Millam, J. M.; Klene, M.; Knox, J. E.; Cross, J. B.; Bakken, V.; Adamo, C.; Jaramillo, J.; Gomperts, R.; Stratmann, R. E.; Yazyev, O.; Austin, A. J.; Cammi, R.; Pomelli, C.; Ochterski, J. W.; Martin, R. L.; Morokuma, K.; Zakrzewski, V. G.; Voth, G. A.; Salvador, P.; Dannenberg, J. J.; Dapprich, S.; Daniels, A. D.; Farkas, Ö.; Foresman, J. B.; Ortiz, J. V.; Cioslowski, J.; Fox, D. J. Gaussian, Inc., Wallingford CT, 2009.

(a) Becke, A. D. J. Chem. Phys. 1993, 98, 5648-5652. (b) Miehlich, B.; Savin, A.; Stoll, H.; Preuss, H. Chem. Phys. Lett. 1989, 157, 200-206. (c) Lee, C.; Yang, W.; Parr, G. Phys. Rev. B 1988, 37, 785-789.

3 Hehre, W. J.; Radom, L.; Schleyer, P. v. R.; Pople, J. A., Ab Initio Molecular Orbital Theory. John Wiley \& Sons, New York, 1986.

Parr, R. G.; Yang, W. Density Functional Theory of Atoms and Molecules, Oxford University Press, New York, 1989.

5 (a) Haeusermann, U.; Dolg, M.; Stoll, H.; Preuss, H. Mol. Phys. 1993, 78, 1211-1224. (b) Kuechle, W.; Dolg, M.; Stoll, H.; Preuss, H. J. Chem. Phys. 1994, 100, 7535-7542. (c) Leininger, T.; Nicklass, A.; Stoll, H.; Dolg, M.; Schwerdtfeger, P. J. Chem. Phys. 1996, 105, 1052-1059. 
6 (a) Ditchfield, R.; Hehre, W. J.; Pople, J. A. J. Chem. Phys. 1971, 54, 724-728; (b) Hehre, W. J.; Ditchfield, R.; Pople, J. A. J. Chem. Phys. 1972, 56, 2257-2261; (c) Hariharan, P. C.; Pople, J. A. Mol. Phys. 1974, 27, 209214; (d) Gordon, M. S. Chem. Phys. Lett. 1980, 76, 163-168; (e) Hariharan, P. C.; Pople, J. A. Theor. Chim. Acta 1973, 28, 213-222. 